\title{
Fator de resposta produtiva de mombaça e marandu ao déficit hídrico
}

\author{
Mircéia Angele MOMBACH${ }^{1}$, Bruno Carneiro PEDREIRA ${ }^{2 *}$, Mariely Lopes SANTOS ${ }^{3}$, \\ Luciano Silva CABRAL ${ }^{1}$, Carolina Silva Alves SANTOS ${ }^{1}$, Ana Mayra Pereira SILVA ${ }^{1}$ \\ ${ }^{1}$ Universidade Federal de Mato Grosso, Cuiabá, MT, Brasil. \\ ${ }^{2}$ Embrapa Agrossilvipastoril, Sinop, MT, Brasil. \\ ${ }^{3}$ Escola Superior de Agricultura Luiz de Queiroz, Universidade de São Paulo, Piracicaba, SP, Brasil. \\ E-mail: bruno.pedreira@embrapa.br
}

\begin{abstract}
Recebido em novembro/2018; Aceito em junho/2019.
RESUMO: O objetivo com este estudo foi compreender o efeito da disponibilidade hídrica sobre a produtividade no capim-mombaça [Megathyrsus maximus (Jacq.) B. K. Simon \& S. W. L. Jacobs] e capim-marandu [Brachiaria brizantha (Hochst. ex A. Rich.) R. D. Webster], determinando o coeficiente de resposta ao déficit hídrico (ky). $\mathrm{O}$ experimento foi avaliado em delineamento de blocos ao acaso, com duas forragens e três repetições. O período experimental correspondeu a doze ciclos de crescimento com intervalos fixos de colheita (28 dias para a estação chuvosa e 42 dias para a estação seca). Os ky foram determinados com base nos dados de produção real e potencial das forrageiras, bem como nos dados de evapotranspiração real e máxima. Para ambas as forragens, o acúmulo de forragem (AF) foi maior no outono e menor no inverno $\left(5310\right.$ e $626 \mathrm{~kg}$ de matéria seca ha ${ }^{-1}$, respectivamente). Na média das quatro estações, o AF para capim-marandu foi 21,3\% maior que o capimmombaça (3344 vs. $2756 \mathrm{~kg}$ matéria seca ha ${ }^{-1}$, respectivamente). Para o capim-mombaça, o ky foi de 1,05, indicando sensibilidade média a alta ao déficit hídrico. No entanto, o ky foi de 0,63 para o capim-marandu, o que representa baixa sensibilidade ao déficit de água do solo.
\end{abstract}

Palavras-chave: Brachiaria; coeficiente de sensibilidade; Megathyrsus; tolerância a seca.

\section{Yield response factor of mombaça guinea grass and marandu palisade grass to water deficit}

\begin{abstract}
The objective with this research was to understand the effect of water availability on Mombaça guinea grass [Megathyrsus maximus (Jacq.) B. K. Simon \& S. W. L. Jacobs] and Marandu palisade grass [Brachiaria brizantha (Hochst. ex A. Rich.) R. D. Webster] productivity, determining the yield response factor to water deficit (ky). The experiment was evaluated in a randomized block design with two forages and three replicates. The experimental period corresponded to twelve growth cycles with fixed harvest intervals (28 days for the rainy season and 42 days for the dry season). The ky were determinate based on the data of real and potential yield of grasses, as well as the data of real and maximum evapotranspiration. To both grasses, herbage accumulation (HA) was greatest in the autumn and leats in the winter $\left(5310\right.$ and $626 \mathrm{~kg}$ dry matter ha ${ }^{-1}$, respectively). On average of the four seasons, the HA for Marandu palisade grass was $21.3 \%$ higher than Mombaça guinea grass (3344 vs $2756 \mathrm{~kg}$ dry matter ha ${ }^{-1}$, respectively). For Mombaça guinea grass, ky was 1.05 , indicating medium to high sensitivity to water deficit. However, the ky was 0.63 for Marandu palisade grass, which represents low sensitivity to soil water deficit.
\end{abstract}

Keywords: Brachiaria; drought tolerance; Megathyrsus; sensitivity coefficient.

\section{INTRODUÇÃO}

A maior parte dos sistemas de produção de bovinos no Brasil tem nas pastagens a principal fonte de alimento, o que evidencia a importância e a necessidade pela busca de práticas de manejo que resultem em maior eficiência (CARVALHO et al., 2017).

Para o crescimento das plantas forrageiras são necessários diversos fatores como luz, temperatura, água e nutrientes (MARTINS et al., 2015). Sempre que a disponibilidade de um ou mais desses fatores é insuficiente, o crescimento das plantas é limitado (TAIZ; ZEIGER, 2013), o que pode resultar em desequilíbrio entre oferta e demanda de alimento e, consequentemente, menor desempenho animal. Entre os fatores mencionados, a disponibilidade hídrica é uma das mais importantes, pois afeta diretamente a produtividade das culturas (CAVALCANTE et al., 2009; FERRARI et al., 2015; SILVA et al., 2014).

Neste sentido, entender a influência da disponibilidade de água sobre a produtividade da cultura é uma condição essencial. Quando há um alto déficit hídrico, que pode ocorrer de forma contínua durante todo o período de crescimento da cultura ou durante um estágio fenológico específico (DOORENBOS; KASSAM, 1979), a produtividade potencial será limitada. A magnitude da disponibilidade de água pode ser quantificada pela determinação do coeficiente ky, que é considerado uma medida da sensibilidade da cultura ao déficit hídrico (SILVA et al., 2014).

Este coeficiente baseia-se na relação entre a produtividade da cultura e o suprimento de água. De um lado, há a necessidade de água para a cultura e os efeitos do déficit 
hídrico e, do outro, os rendimentos de produção da cultura (KABOOSI; KAVEH, 2012). A quantificação deste coeficiente fornece um meio eficaz de simular a produtividade da cultura em resposta ao déficit hídrico para uma determinada região (GREAVES; WANG, 2017).

Nesse contexto, o conhecimento do efeito do déficit hídrico sobre a produção é primordial para definir o manejo mais adequado para uma cultura (FERRARI et al., 2015). Esse conhecimento pode contribuir para a escolha de espécies forrageiras mais adaptadas às variações climáticas (ANDRADE et al., 2014; PEZZOPANE et al., 2017). Além disso, pode contribuir para melhorar a eficiência de sistemas de produção em pastagens e, consequentemente, com os resultados de produção animal. Assim, o objetivo com este estudo foi compreender o efeito da disponibilidade hídrica sobre a produtividade no capim-mombaça e capim-marandu, determinando o coeficiente de resposta ao déficit hídrico (ky).

\section{MATERIAL E MÉTODOS}

$\mathrm{O}$ experimento foi realizado na área experimental da Embrapa Agrossilvipastoril, Sinop, Mato Grosso, Brasil (latitude $-11^{\circ} 51^{\prime} \mathrm{N}$; longitude $55^{\circ} 36^{\prime} \mathrm{O}$ e altitude de $370 \mathrm{~m}$ ). O clima da região foi classificado segundo os critérios de Köppen, como Am - clima de monções, com alternância entre a estação das chuvas e estação seca bem definida (ALVARES et al., 2013). O histórico de temperatura média, mínima e máxima anuais entre 2004 e 2013 foram, respectivamente, de 25,20 e $34^{\circ} \mathrm{C}$, com umidade relativa de $73,5 \%$ e precipitação anual de 1800 a $2300 \mathrm{~mm}$ (ALVARES et al., 2013; Instituto Nacional De Metereologia - INMET, 2016).

O solo foi classificado como Rhodic Hapludox (SOIL SURVEY STAFF, 2014), de relevo levemente inclinado, com textura argilosa $\left(586 \mathrm{~g} / \mathrm{dm}^{3}\right)$ e densidade de $1,22 \mathrm{~g} \mathrm{~cm}^{-3}$. A média das características químicas do solo (camada $0-20 \mathrm{~cm}$ ) no início do experimento foram: $\mathrm{pH}=5,00\left(\mathrm{CaCl}_{2}\right)$; matéria orgânica $=28,23 \mathrm{~g} \mathrm{~kg}^{-1} ; \mathrm{P}=2,87 \mathrm{mg} \mathrm{dm}^{-3} ; \mathrm{K}=98 \mathrm{mg} \mathrm{dm}^{-3}$; $\mathrm{Ca}=1,59 \mathrm{cmol} \mathrm{dm}^{-3} ; \mathrm{Mg}=0,81 \mathrm{cmol} \mathrm{dm}^{-3} ; \mathrm{H}+\mathrm{Al}=5,12 \mathrm{cmol}$ $\mathrm{dm}^{-3}$; soma de bases $=2,65 \mathrm{cmol} \mathrm{dm}^{-3}$, capacidade de troca catiônica $(\mathrm{pH} 7,0)=7,77 \mathrm{cmol} \mathrm{dm}^{-3}$; e saturação por bases $=$ $34,08 \%$. A aplicação de calagem foi realizada com meta de saturação por bases de 70\%, conforme Boletim Técnico 100 (RAIJ et al., 2001), de modo a garantir que não houvesse limitação de crescimento para ambas as cultivares.

A semeadura do capim-marandu e capim-mombaça ocorreram em 25 de novembro de 2014. No dia 4 de dezembro, $100 \mathrm{~kg} \mathrm{ha}^{-1}$ de $\mathrm{P}_{2} \mathrm{O}_{5}$ foi aplicado na forma de superfosfato simples em cobertura, e no dia 15 de dezembro de 2014 foi realizado a primeira calagem. A segunda calagem ocorreu no dia 18 de setembro de 2015, concomitante com as aplicações de $100 \mathrm{~kg} \mathrm{ha}^{-1}$ de $\mathrm{P}_{2} \mathrm{O}_{5}$ na forma de superfosfato simples e de $60 \mathrm{~kg} \mathrm{ha}^{-1}$ de fertilizante granulado micro FTE.

O período experimental foi de 383 dias, compreendidos entre 21 março de 2015 a 07 de abril de 2016, totalizando 12 ciclos de crescimento simulando lotação intermitente. Um corte de uniformização dos capins foi feito 28 dias antes do primeiro ciclo. O delineamento foi em blocos completos casualizados com 3 repetições por bloco para cada cultivar, totalizando 12 unidades experimentais que corresponderam a parcelas de $32 \mathrm{~m}^{2}(8 \times 4 \mathrm{~m})$, espaçadas por 2 metros entre parcelas e 2 metros entre blocos.

As avaliações de acúmulo de forragem (AF; $\mathrm{kg} \mathrm{ha}^{-1}$ ) foram realizadas a cada 28 dias entre os meses de novembro e abril, e a cada 42 dias entre maio e outubro. Em cada ciclo, a forragem foi colhida em dois locais por parcela $(0,5 \times 1,0 \mathrm{~m})$, deixando-se um resíduo de 40 e $15 \mathrm{~cm}$ para o capim-mombaça e capim-marandu, respectivamente. Cada amostra foi subamostrada, pesada e levada à estufa de circulação de ar forçada a $55^{\circ} \mathrm{C}$ por $72 \mathrm{~h}$. O AF em cada ciclo foi dividido pelo respectivo número de dias em rebrotação e então utilizados para definir o AF nas 4 estações do ano (outono, inverno, primavera de 2015 e verão de 2015/2016).

Os dados meteorológicos do período experimental foram registrados em uma estação automática localizada a $500 \mathrm{~m}$ da área experimental. Para a estimativa da disponibilidade de água, utilizou-se a metodologia proposta por Thonrthwaite; Mather (1955) de balanço hídrico, em intervalos regulares de $10 \pm 2$ dias em função dos ajustes para os intervalos de corte (Figura 1). A evapotranspiração de referência (ETo) foi estimada pelo método de Penman-Monteith (ALLEN et al., 1998). Os valores do coeficiente da cultura (kc) para capimmombaça foram determinados a partir de equações propostas por Rodrigues et al. (2009). Nos ciclos de novembro a abril (período chuvoso) foi utilizado o kc de 1,51 e para os ciclos de maio a outubro (período seco) foi utilizado o kc de 1,60. Para o capim-marandu adotou-se o kc médio de 1,19 para todo o período, como proposto por Sanches et al. (2017) após estabilização da cultura (48-56 dias após emergência) e independente do intervalo de corte.

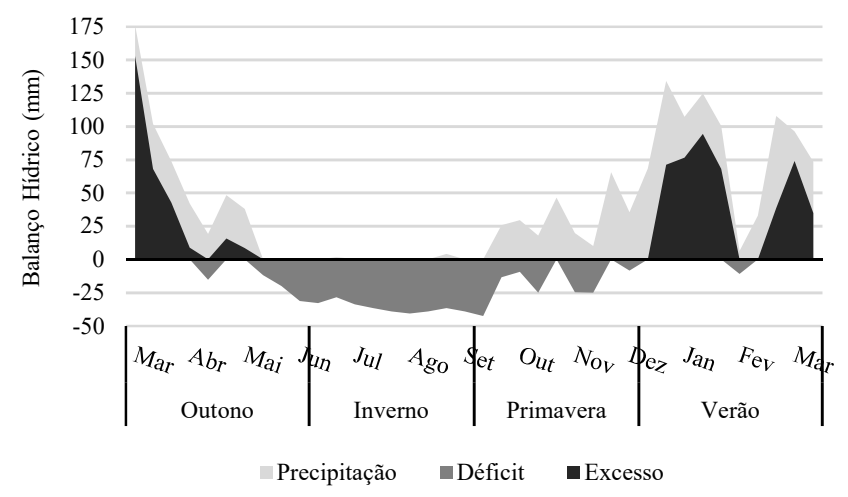

Figura 1. Precipitação, déficit e excesso de água durante o período experimental (Thornthwaite; Mather, 1955).

Figure 1. Rainfall, deficit and water excess during the experimental period (Thornthwaite; Mather, 1955).

Para o cálculo do coeficiente de resposta da cultura ao déficit hídrico (ky), utilizou-se o modelo descrito por Doorenbos; Kassam (1979) (Equação 1):

$$
(1-Y r / Y p)=k y \cdot(1-E T r / E T m)
$$

em que: Yr: rendimento real obtido no experimento, em $\mathrm{kg} \mathrm{ha}^{-1}$; Yp: rendimento potencial, em $\mathrm{kg} \mathrm{ha}^{-1}$; ky: coeficiente de resposta da cultura; ETr: evapotranspiração real, em $\mathrm{mm}$; ETm: evapotranspiração máxima, em mm.

O rendimento potencial de produção (Yp) da forragem por ciclo de crescimento foi determinado utilizando dados de Carnevalli et al. (2006) para o capim-mombaça e de Flores et al. (2008) para o capim-marandu, considerando a altura de resíduo de 40 e $15 \mathrm{~cm}$, respectivamente para cada capim.

$\mathrm{O}$ valor da capacidade de água disponível no solo (CAD) foi estabelecido pela capacidade média de água livre, que para solos argilosos assumiu-se como de $2 \mathrm{~mm} \mathrm{~cm}^{-1}$ e pela profundidade específica do sistema radicular. Para o capim- 
mombaça, o comprimento do sistema radicular utilizado foi de $22,54 \mathrm{~cm}$ e para o capim-marandu de $43,14 \mathrm{~cm}$ (CUNHA et al., 2010).

Os dados foram analisados utilizando o método de modelos mistos com estrutura paramétrica especial na matriz de covariância, com medidas repetidas no tempo, por meio do procedimento MIXED do software estatístico SAS (Statistical Analysis System, version 9.4). Cultivar e estação do ano foram considerados como efeitos fixos e o bloco como efeito aleatório (LITTELL et al., 2006). Para a escolha da matriz de covariância foi usado o critério de informação de Akaike (Akaike information criterion, AIC) (WOLFINGER, 1993). As médias dos tratamentos foram estimadas utilizando o LSMEANS e a comparação entre elas foi realizada por meio da probabilidade da diferença "PDIFF" $(\mathrm{p}<0.05)$.

Para avaliar a relação entre o déficit na evapotranspiração relativa (DER) e o AF foram feitas análises de correlação pelo procedimento CORR $(\mathrm{p}<0.05)$ e a determinação das equações de regressão pelo procedimento REG. Ambos os procedimentos foram feitos utilizando o software estatístico SAS (Statistical Analysis System, version 9.4).

\section{RESULTADOS}

Durante o período experimental, a menor temperatura foi de $14,4^{\circ} \mathrm{C}$ no mês de julho/2015 e a maior no mês de outubro/2015 $\left(39,1^{\circ} \mathrm{C}\right.$, Figura 2). A radiação global e a umidade relativa média variaram entre 5,52 e $27,23 \mathrm{MJ} / \mathrm{m}^{2} /$ dia e 41 e $89 \%$, respectivamente.

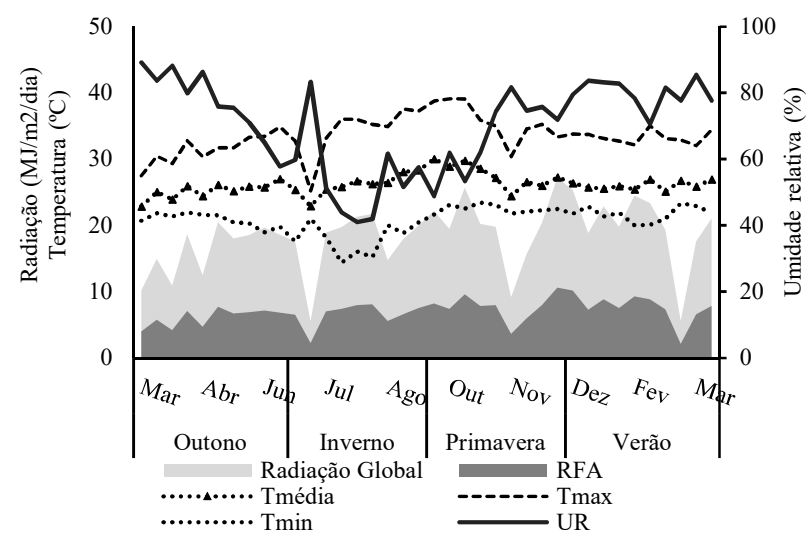

Figura 2. Temperatura média (Tmédia), máxima (Tmax) e mínima (Tmin), radiação global, umidade relativa (UR) e radiação fotossinteticamente ativa (RFA) durante o período experimental.

Figure 2. Mean (Tmean), maximum (Tmax) and minimum (Tmin) temperature, global radiation, relative humidity $(\mathrm{RH})$ and photosynthetically active radiation (PAR) during the experimental period.

Não houve efeito de interação cultivar x época do ano $(\mathrm{p}=0,0712)$ para a acúmulo de forragem $(\mathrm{AF})$, embora haja efeito de cultivar $(\mathrm{p}=0,0154)$ e estação do ano $(\mathrm{p}<0,0001)$. Na média das quatro estações, o AF para capim-marandu foi $21,3 \%$ maior que o capim-mombaça, uma diferença de $587 \mathrm{~kg}$ ha $^{-1}$ (Tabela 1).

Independente do período de avaliação, os maiores AF foram observados no outono, independente da cultivar, com acúmulo 44\% superior ao registrado no verão, com o segundo maior AF. Enquanto os menores AF foram observados durante o inverno.

$\mathrm{O}$ efeito negativo do déficit hídrico no solo sobre a produtividade da forrageira, avaliado durante o período experimental, foi mais acentuado nos ciclos 4,5 e 6 para o capim-mombaça (Tabela 2). Nesses ciclos, a redução no acúmulo de forragem foi de 91, 100 e 94\%, respectivamente, em comparação à produção potencial deste capim com base nos trabalhos científicos (1-Yr/Yp). As maiores produções (Yr) foram mensuradas nos ciclos 1, 2, 3 e 10, sem, no entanto, superar a produção potencial estimada na literatura (Yp).

Tabela 1. Acúmulo de forragem ( $\left.\mathrm{kg} \mathrm{MS} \mathrm{ha}{ }^{-1}\right)$ do capim-mombaça e capim-marandu ao longo das estações do ano.

Table 1. Herbage accumulation $\left(\mathrm{kg} \mathrm{DM} \mathrm{ha}{ }^{-1}\right)$ of Mombaça guinea grass and Marandu palisade grass through the seasons.

\begin{tabular}{lccccc}
\multirow{2}{*}{ Cultivar } & \multicolumn{5}{c}{ Estação } \\
\cline { 2 - 5 } & Outono & Inverno & Primavera & Verão & \\
\hline Mombaça & 5633 & 308 & 1859 & 3225 & 2756B \\
Marandu & 4986 & 944 & 3327 & 4119 & 3344A \\
\hdashline Média & $5310 \mathrm{a}$ & $626 \mathrm{~d}$ & $2592 \mathrm{c}$ & $3672 \mathrm{~b}$ & \\
\hline
\end{tabular}

Médias seguidas pela mesma letra minúscula na linha e maiúscula na coluna não diferem no teste de significância a $5 \%$ de probabilidade de diferença (pdiff).

Assim como no capim-mombaça, os ciclos 4, 5 e 6 registraram reduções acentuadas no acúmulo de forragem do capim-marandu (Yr) em relação a produção potencial (Yp) deste capim. Isso representou uma redução média de $80 \% \mathrm{em}$ relação a produção potencial de forragem do capim-marandu (Tabela 3). Contudo, nos ciclos 3, 8, 9 e 12, o acúmulo de foragem do capim-marandu superou em 43, 42, 31 e 1\%, respectivamente, a produção potencial estimada por ciclo de crescimento.

Tabela 2. Evapotranspiração real (ETr, mm) e máxima (ETm, mm), déficit de evapotranspiração relativa - DER (1 - ETr/ETm), produção de forragem real (Yr, t/ha) e potencial (Yp, t/ha) e o declínio na produção relativa - DPR (1 - Yr/Yp) em pastos de capim-mombaça. Table 2. Real evapotranspiration (ETr, $\mathrm{mm}$ ) and maximum (ETm, $\mathrm{mm})$, relative evapotranspiration deficit - DER (1 - ETr/ETm), real production yield (Yr, t/ha) and potential (Yp, t/ha) and decline in relative production yield - DPR $(1-\mathrm{Yr} / \mathrm{Ym})$ in pasture of Mombaça guinea grass.

\begin{tabular}{cccccccc}
\hline Ciclo & Período $^{1}$ & Etr & Etm & DER & Yr & Yp $^{2}$ & DPR \\
\hline 1 & $13 / 03-09 / 04$ & 29,46 & 42,72 & 0,310 & 2192 & 2200 & 0,004 \\
2 & $10 / 04-07 / 05$ & 28,32 & 48,55 & 0,413 & 2037 & 2200 & 0,07 \\
3 & $08 / 05-18 / 06$ & 18,52 & 54,98 & 0,653 & 1665 & 2200 & 0,24 \\
4 & $19 / 06-30 / 07$ & 0,53 & 53,50 & 0,989 & 198 & 2200 & 0,91 \\
5 & $31 / 07-10 / 09$ & 1,02 & 63,95 & 0,984 & 0 & 2200 & 1,00 \\
6 & $11 / 09-22 / 10$ & 13,78 & 63,89 & 0,779 & 138 & 2200 & 0,94 \\
7 & $23 / 10-19 / 11$ & 28,03 & 58,14 & 0,477 & 516 & 2200 & 0,76 \\
8 & $20 / 11-18 / 12$ & 36,92 & 57,96 & 0,385 & 1192 & 2200 & 0,46 \\
9 & $19 / 12-15 / 01$ & 39,02 & 56,57 & 0,310 & 956 & 2200 & 0,56 \\
10 & $16 / 01-12 / 02$ & 31,21 & 50,58 & 0,371 & 1320 & 2200 & 0,40 \\
11 & $13 / 02-10 / 03$ & 33,28 & 45,22 & 0,273 & 919 & 2200 & 0,58 \\
12 & $11 / 03-07 / 04$ & 37,18 & 53,91 & 0,310 & 1108 & 2200 & 0,50 \\
\hline
\end{tabular}

${ }^{1}$ Correspondente ao ciclo de crescimento; ${ }^{2}$ Carnevalli et al. (2006).

Para ambos as forrageiras, os maiores valores de déficit de evapotranspiração relativa (1 - ETr/ETm) foram observados nos ciclos 4, 5 e 6 (Tabela 2 e 3) com as menores produção reais (Yr) nestes ciclos. Para o capim-mombaça, o ky médio foi de $1,05(0,01-2,23)$ e para o capim-marandu de $0,63(0-$ $2,05)$.

Os modelos correlacionando à redução de produtividade e $o$ déficit de evapotranspiração relativa foram significativos ( $\mathrm{p}$ $<0,05)$ para ambos os capins. Em relação ao capim-mombaça, $44 \%$ das variações da redução de produtividade relativa é explicada pelo déficit de evapotranspiração, enquanto que para 
o capim-marandu esse percentual é de 76\% (Figura 3). A inclinação na curva indica um aumento na queda dos rendimentos relativos (produtividade) com o incremento no déficit de evapotranspiração relativa.

Tabela 3. Evapotranspiração real (ETr, mm) e máxima (ETm, mm), déficit de evapotranspiração relativa - DER (1 - ETr/ETm), produção de forragem real (Yr, t/ha) e potencial (Yp, t/ha) e o declínio na produção relativa - DPR (1 - Yr/Yp) em pastos de capim-marandu. Table 3. Real evapotranspiration (ETr, $\mathrm{mm}$ ) and maximum (ETm, $\mathrm{mm})$, relative evapotranspiration deficit - DER (1 - ETr/ETm), real production yield $(\mathrm{Yr}, \mathrm{t} / \mathrm{ha}$ ) and potential $(\mathrm{Yp}, \mathrm{t} / \mathrm{ha})$ and decline in relative production yield - DPR (1 - Yr/Ym) in pasture of Marandu palisade grass.

\begin{tabular}{cccccccc} 
Ciclo & Período $^{1}$ & Etr & Etm & DER & Yr & Yp $^{2}$ & DPR \\
\hline 1 & $13 / 03-09 / 04$ & 29,46 & 35,06 & 0,160 & 1145 & 1470 & 0,22 \\
2 & $10 / 04-07 / 05$ & 28,32 & 39,85 & 0,285 & 1382 & 1470 & 0,06 \\
3 & $08 / 05-18 / 06$ & 27,64 & 40,89 & 0,319 & 2107 & 1470 & 0,00 \\
4 & $19 / 06-30 / 07$ & 2,33 & 39,79 & 0,939 & 339 & 1470 & 0,77 \\
5 & $31 / 07-10 / 09$ & 1,02 & 47,57 & 0,979 & 293 & 1470 & 0,80 \\
6 & $11 / 09-22 / 10$ & 13,78 & 47,52 & 0,703 & 251 & 1470 & 0,83 \\
7 & $23 / 10-19 / 11$ & 28,03 & 47,71 & 0,362 & 971 & 1470 & 0,34 \\
8 & $20 / 11-18 / 12$ & 36,92 & 47,57 & 0,250 & 2086 & 1470 & 0,00 \\
9 & $19 / 12-15 / 01$ & 39,02 & 46,43 & 0,160 & 1926 & 1470 & 0,00 \\
10 & $16 / 01-12 / 02$ & 32,78 & 41,51 & 0,202 & 905 & 1470 & 0,39 \\
11 & $13 / 02-10 / 03$ & 36,59 & 37,11 & 0,043 & 1304 & 1470 & 0,11 \\
12 & $11 / 03-07 / 04$ & 37,18 & 44,24 & 0,160 & 1487 & 1470 & 0,00 \\
\hline
\end{tabular}

${ }^{1}$ Correspondente ao ciclo de crescimento; ${ }^{2}$ Flores et al. (2008).

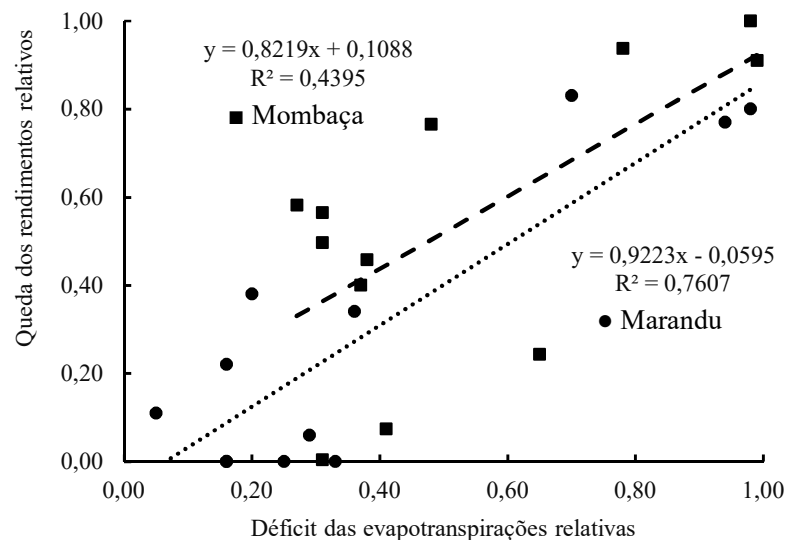

Figura 3. Relação entre o déficit de evapotranspiração relativa e a queda dos rendimentos relativos na produção capim-mombaça e capim-marandu.

Figure 3. Relation between the relative evapotranspiration deficit and relative yield decline in production of the Mombaça guinea grass and Marandu palisade grass.

\section{DISCUSSÃO}

A redução de $88 \%$ no AF no inverno é consequência dos baixos índices pluviométricos que resultaram em uma deficiência hídrica acumulada acima de $325 \mathrm{~mm}$ (Figura 1). O suprimento de água para qualquer cultura é variável em função de todas as características que definem a interação entre o sistema solo-planta-atmosfera. Nesse sentido, a medida em que ocorre redução da disponibilidade hídrica no solo, a água remanescente fica fortemente retida pelos sólidos no solo, especialmente nos poros menores, o que reduz a absorção de água pela planta (SANTOS; CARLESSO, 1998).

A principal resposta das plantas ao déficit hídrico é a redução no crescimento foliar, em virtude da diminuição no potencial de água na folha que proporciona o fechamento estomático e limita a entrada de $\mathrm{CO}_{2} \mathrm{e}$, consequentemente, a taxa de fotossíntese (OSAKABE et al., 2014). Segundo Cavalcante et al. (2009), durante o período de seca ocorrem as maiores reduções no AF em função da baixa atividade fisiológica da planta decorrente do déficit hídrico.

É importante ressaltar que no final do verão e o começo do outono foram registradas altas precipitações, o que resultou em excedente hídrico no solo (Figura 1). Esse fato contribuiu para que os maiores registros de produtividade fossem alcançados em relação às demais estações do ano (Tabela 1). Melo et al. (2009) também observaram maior acúmulo de forragem em Mombaça e Marandu quando o solo apresentava os maiores índices de disponibilidade hídrica.

As baixas temperaturas registradas durante a estação de inverno se aproximam da temperatura basal inferior de $11^{\circ} \mathrm{C}$ para o capim-mombaça (MORENO et al., 2014) e para o capim-marandu (PEZZOPANE et al., 2018). Segundo estes autores, a temperatura basal inferior é a temperatura que limita os processos que resultam no AF, tornando-o nulo, desprezível ou mínimo. As baixas temperaturas associada a redução na disponibilidade hídrica no solo na estação do inverno (Figura 1) explicam os menores AF observados nesse período (Tabela 1) e as menores rendimentos reais (Yr) para o capim-mombaça e marandu (Tabela 2 e 3). No entanto, o efeito do fotoperíodo e de disponibilidade de radiação fotossinteticamente ativa não são apontados como fatores limitantes ao AF nessa região (NASCIMENTO et al., 2019; GOMES et al., 2019).

Em muitos locais no Brasil, a estacionalidade da produção de forragem, imposta pelas condições meteorológicas, ocorre de acordo com distribuição espacial e temporal das chuvas. Dessa forma, períodos de estiagem prolongados afetam os processos fotossintéticos (MARTINS et al., 2015) e, consequentemente, reduzem a produção de forragem (RODRIGUES et al., 2009). Quanto mais longo for o período de estiagem, pior são as consequências para os sistemas de produção animal em pastagens. Mattos et al. (2005) verificaram reduções de $88 \%$ no AF em capins do gênero Brachiaria durante o período de déficit hídrico no solo. Este valor foi maior do que o observado neste experimento (Tabela 3). No entanto, de todas as espécies avaliadas, a $B$. brizantha cv. Marandu apresentou o maior AF durante o período de déficit hídrico no solo.

Com o entendimento da relação entre a redução de produtividade relativa e o déficit de evapotranspiração relativa é possível obter o coeficiente de resposta ao déficit hídrico (ky). Esse coeficiente relaciona a magnitude do efeito do déficit hídrico sobre a produtividade. De acordo com Dorenboos; Kassam (1994), as culturas são classificadas quanto a sua sensibilidade ao déficit hídrico como baixo (ky < $0,85)$, baixo/média $(0,85<\mathrm{ky}<1,00)$, médio/alto $(1,00<\mathrm{ky}$ $<1,15)$ e alto $(\mathrm{ky}>1,15)$.

O ky obtido para o capim-mombaça demonstra que está forrageira apresenta média a alta sensibilidade ao déficit hídrico, enquanto o capim-marandu possui baixa sensibilidade. Isto implica em maior resistência do capimmarandu em regiões com acentuada diferença no regime pluviométrico ao longo do ano. Pezzopane et al. (2018) verificaram que o limite inferior de água no solo é $82 \%$ para o capim-marandu. Sob estas condições, está forrageira não apresenta perda de produtividade. Isso indica que mesmo quando a capacidade de campo é reduzida em 18\%, a produtividade para esta cultivar pode não ser afetada. Uma das justificativas é o maior comprimento de raiz desta forrageira em detrimento ao capim-mombaça (CUNHA et al., 2010). 
Avaliando o efeito do déficit hídrico entre as espécies de Brachiaria brizantha, Pezzopane et al. (2015) observaram que a cultivar Marandu apresenta maior tolerância ao déficit hídrico. Estes autores observaram um aumento de $56 \mathrm{~cm}^{2} /$ vaso de área foliar, com menor taxa de alongamento, para a cultivar Marandu em detrimento aos demais genótipos de $B$. brizantha avaliados sob déficit hídrico. A redução na expansão da taxa foliar é uma resposta precoce adaptativa ao déficit hídrico devido ao decréscimo na pressão de turgor (TAIZ; ZAIGER 2013). Ademais, a área foliar é uma característica relevante no desenvolvimento inicial de gramíneas por ser uma fonte de fotoassimilados para o desenvolvimento das raízes, o que desempenha importante papel na resistência ao déficit hídrico (BONFIM-SILVA et al., 2011).

A compreensão dessas variações ao longo do ano e entre os anos é marcadamente importante para o planejamento do sistema de produção, o que permite melhorias na estimativa do crescimento e acúmulo de forragens tropicais (ANDRADE et al., 2016).

Espécies forrageiras mais resistentes ao déficit hídrico e, ou que utilizam de maneira mais eficiente a água são alternativas para adaptar os sistemas de produção animal em pastagens às mudanças climáticas globais (ANDRADE et al., 2014; PEZZOPANE et al., 2017; SANTOS et al., 2013). Eventos de seca estão entre os fatores abióticos que periodicamente impõem desafios em espécies forrageiras de clima tropical, afetando aspectos fisiológicos da planta e, portanto, a produtividade (BELONI et al., 2018).

Em estudo avaliando a eficiência de uso de água por espécies forrageiras tropicais (C4) e temperadas (C3), Beloni et al. (2018) verificaram que a as espécies de clima tropical são mais eficientes em utilizar a água. Dentre as espécies avaliadas, a cultivar Marandu apresentou a maior eficiência de utilização $\left(3,6 \times 10^{-3} \mathrm{~g} \mathrm{MS}\right.$ produzida/g $\mathrm{H}_{2} \mathrm{O}$ utilizada). Isso implica em maior potencial de produção dessa forragem que pode ser considerada uma alternativa para as mudanças significativas na precipitação. Assim como, para o aumento das temperaturas máxima e mínima que são previstas em alguns cenários de estudos no Brasil (ANDRADE et al., 2014).

Embora o entendimento sobre mudanças climáticas possa sugerir a busca por uma cultivar mais resiliente, a utilização de várias espécies na mesma propriedade é muito importante para reduzir os riscos de colapso nos sistemas de produção animal em pastagens (PEDREIRA; PEDREIRA, 2014).

\section{CONCLUSÕES}

O capim-marandu pode ser considerado uma cultivar com melhor potencial de adaptação às mudanças climáticas, com alta produção de forragem e baixa sensibilidade ao déficit hídrico.

\section{REFERÊNCIAS}

ALLEN, R. G.; PEREIRA, L. S.; RAES, D.; SMITH, M. Crop evapotranspiration: guidelines for computing crop water requirements. Rome: FAO, 1998, 300 p. (FAO. Irrigation and Drainage Paper, 56).

ALVARES, C. A.; STAPE, J. L.; SENTELHAS, P. C.; DE MORAES, G.; LEONARDO, J.; SPAROVEK, G. Köppen's climate classification map for Brazil. Meteorologische Zeitschrift, Berlin, v. 22, n. 6, p. 711728, 2013. DOI: http://dx.doi.org/10.1127/0941$2948 / 2013 / 0507$
ANDRADE, A. S.; SANTOS, P. M.; PEZZOPANE, J. R. M.; BETTIOL, G. M.; EVANGELISTA, S. R. M. Climate change and future scenarios for palisade grass production in the state of São Paulo, Brazil. Pesquisa Agropecuária Brasileira, Brasília, v. 49, n. 10, p. 745-753, 2014. DOI: http://dx.doi.org/10.1590/S0100-204X2014001000001

ANDRADE, A. S.; SANTOS, P. M.; PEZZOPANE, J. R. M.; ARAUJO, L. C.; PEDREIRA, B. C.; PEDREIRA, C. G. S.; MARIN, F. R.; LARA, M. A. S. Simulating tropical forage growth and biomass accumulation: an overview of model development and application. Grass and Forage Science, Oxford, v. 71, n. 1, p. 54-64, 2016. DOI: http://dx.doi.org/10.1111/gfs.12177

BELONI, T.; SANTOS, P. M.; ROVADOSCKI, G. A.; BALACHOWSKI, J.; VOLAIRE, F. Large variability in drought survival among Urocloa spp. cultivars. Grass and Forage Science, Oxford, v. 73, n. 4, p. 1-11, 2018. DOI: http://dx.doi.org/10.1111/gfs.12380

BONFIM-SILVA, E. M.; SILVA, T. J. A.; CABRAL, C. E. A.; KROTH, B. E.; REZENDE, D. Desenvolvimento inicial de gramíneas submetidas ao estresse hídrico. Revista Caatinga, Mossoró, v. 24, n. 2, p. 180-186, 2011. CARNEVALli, R. A.; SILVA, S. C.; BUENO, A. A. O.; UEBELE, M. C.; BUENO, F. O.; HODGSON, J.; SILVA, G. N.; MORAIS, J. P. G. Herbage production and grazing losses in Panicum maximum cv. Mombaça under four grazing managements. Tropical Grasslands, Brisbane, v. 40, p. 165-176, 2006.

CARVALHO, A. L. S.; MARTUSCELLO, J. Á.; ALMEIDA, O. G.; BRAZ, T. G. S.; CUNHA, D. N. F. V.; JANK, L. Production and qualit of Mombaça grass forage under diferente residual heights. Acta Scientiarium Animal Sciences, Maringá, v. 39, n. 2, p. 143-148, 2017. DOI: http://dx.doi.org/10.4025/actascianimsci.v39i2.34599

CAVALCANTE, A. C. R.; CAVALLINI, M. C.; LIMA, N. R. C. B. Estresse por déficit hídrico em plantas forrageiras. Sobral: Embrapa Caprinos e Ovinos, 2009. 47 p. (Documentos, 89).

CUNHA, F. F.; RAMOS, M. M.; ALENCAR, C. A. B.; MARTINS, C. E.; CÓSER, A. C.; OLIVEIRA, R. A. Sistema radicular de seis gramíneas irrigadas em diferentes adubações nitrogenadas e manejos. Acta Scientiarum. Agronomy, Maringá, v. 32, n. 2, p. 351-357, 2010. DOI: http://dx.doi.org/10.4025/actasciagron.v32i2.1020

DOORENBOS, J.; KASSAM, A. H. Efeito da água no rendimento das culturas. Campina Grande: UFPB, 1994, 306 p. (Estudos FAO: Irrigação e Drenagem, 33)

FERRARI, E.; PAZ, A.; SILVA, A. C. Déficit hídrico no metabolismo da soja em semeaduras antecipadas no Mato Grosso. Nativa, Sinop, v. 3, n. 1, p. 67-77, 2015. DOI: http://dx.doi.org/10.14583/2318-7670.v03n01a12

FLORES, R. S.; EUCLIDES, V. P. B.; ABRÃO, M. P. C.; GALBEIRO, S.; DIFANTE, G. S.; BARBOSA, R. A. Desempenho animal, produção de forragem e características estruturais dos capins marandu e xaraés submetidos a intensidades de pastejo. Revista Brasileira de Zootecnia, v. 37, n. 8, p. 1355-1365, 2008. DOI: http://dx.doi.org/10.1590/S151635982008000800-004

GOMES, F. J.; PEDREIRA, C. G. S.; BOSI, C.; CAVALLI, J.; HOLSCHUCH, S. G.; MOURÃO, G. B.; PEREIRA, D. H.; PEDREIRA, B. C. Shading effects on Marandu palisadegrass in a silvopastoral system: plant morphological and physiological responses. Agronomy 
Journal, Madison, v. 111, n. 5, p. 2332-2340, 2019. DOI: https://dx.doi.org/10.2134/agronj2019.01.0052

GREAVES, G. E.; WANG, Y.-M. Yield response, water productivity, and seasonal water production functions for maize under deficit irrigation water management in southern. Plant Production Science, v. 20, n. 4, p. 353365 , 2017.

DOI https://dx.doi.org/10.1080/1343943X.2017.1365613

KABOOSI, K.; KAVEH, F. Sensitivity analysis of FAO 33 crop water production function. Irrigation Science, New York, v. 30, n. 2, p. 89-100. DOI: http://dx.doi.org/10.1007/s00271-011-0263-7

INMET INSTITUTO NACIONAL DE METEOROLOGIA. Dados Históricos. 2016. Disponível em: $<$ http://www.inmet.gov.br/portal/index.php?r=bdmep/bd mep>. Acesso em: 10 mar 2017.

LITTELl, R. C.; MILLIKEN, G. A.; STROUP, W. W.; WOLFINGER, R. D.; SCHABENBERGER, O. Sas for mixed models. 2. ed. Cary: SAS Institute, 2006, 813 p.

MARTINS, L. E. C.; MONTEIRO, F. A.; PEDREIRA, B. C. Photosynthesis and leaf area of Brachiaria brizantha in response to phosphorus and zinc nutrition. Journal of Plant Nutrition, New York, v. 38, n. 5, p. 754-767, 2015. DOI: https://doi.org/10.1080/01904167.2014.939758

MATTOS, J. L. S.; GOMIDE, J. A.; HUAMAN, C. A. M. Crescimento de espécies de Brachiaria sob déficit hídrico e alagamento a campo. Revista Brasileira de Zootecnia, Viçosa, v. 34, n. 3, p. 755-764, 2005. DOI: http://dx.doi.org/10.1590/S1516-35982005000300006

MELO, J. C.; SANTOS, A. C.; ALMEIDA, J. Á.; MORAIS NETO, L. R. Desenvolvimento e produtividade dos capins mombaça e marandu cultivadas em dois solos típicos do Tocantins com diferentes regimes hídricos. Revista Brasileira de Saúde e Produção Animal, Salvador, v. 10, n. 4, p. 786- 800, 2009

MORENO, L. S. B.; PEDREIRA, C. G. S.; BOOTE, K. J.; ALVES, R. R. Base temperature determination of tropical Panicum spp. grasses and its effects on degree-day-based models. Agricultural and Forest Meteorology, Amsterdam, v. 186, p. 26-33, 2014. DOI: http://dx.doi.org/10.1016/j.agrformet.2013.09.013

NASCIMENTO, H. L. B.; PEDREIRA, B. C.; SOLLENBERGER, L. E.; PEREIRA, D. H.; MAGAlHÃES, C. A. S.; CHIZZOTI, F. H. M. Physiological characteristics and forage accumulation of grazed Marandu palisade grass (Brachiaria brizantha) growing in monoculture and in silvopasture with Eucalyptus urograndis. Crop \& Pasture Science, Victoria, v. 70, n. 4, p. 384-394. DOI: http://dx.doi.org/10.1071/CP18403

OSAKABE, Y.; OSAKABE, K.; SHINOZAKI, K.; TRAN, LAM-S. P. Response of plants to water stress. Frontiers in Plant Science, v. 5, n. 86, p. 1-8, 2014. DOI: http://dx.doi.org/10.3389/fpls.2014.00086

PEDREIRA, C. G. S.; PEDREIRA, B. C. Manejo de pastagens tropicais para a intensificação da pecuária. In: PEDREIRA, B. C.; PEREIRA, D. H.; PINA, D. S.; CARNEVALLI, R. A.; LOPES, L. B. (Eds.) Intensificação da produção animal em pastagens. Sinop: Embrapa Agrossilvipastoril, 2014. p. 83-108.

PEZZOPANE, C. G.; SANTOS, P. M.; CRUZ, P. G.; ALTOÉ, J.; RIBEIRO, F. A.; VALLE, C. B. Estresse por deficiência hídrica em genótipos de Brachiaria brizantha. Ciência
Rural, Santa Maria, v. 45, n. 05, p. 874-876, 2015. DOI: http://dx.doi.org/10.1590/0103-8478cr20130915

PEZZOPANE, J. R. M.; SANTOS, P. M.; EVANGELISTA, S. E. M.; BOSI, C.; CAVALCANTE, A. C. R.; BETTIOL, G. M.; GOMIDE, C. A. M.; PELlEGRINO, C. Q. Panicum maximum cv. Tanzânia: climate trends and regional pasture production in Brazil. Grass and Forage Science, Oxford, v. 72, n. 1, p. 104-117, 2017. DOI: http://dx.doi.org/10.1111/gfs.12229

PEZZOPANE, J. R. M.; SANTOS, P. M.; CRUZ, P. G.; BOSI, C.; SENTELHAS, P. C. An integrated agrometeorological model to simulate Marandu palisade grass productivity. Field Crops Research, Amsterdam, v. 224, p. 13-21, 2018. DOI: https://dx.doi.org/10.1016/j.fcr.2018.04.015

RAIJ, B. V.; ANDRADE, J. C.; CANTARELA, H.; QUAGGIO, J. A. Análise Química para fertilidade de solos tropicais. 1. ed. Campinas: Instituto Agronômico fundação IAC, 2001, 285 p.

RODRIGUES, B. H. N.; BASTOS, E. A.; FERNANDES, P. D.; ANDRADE JÚNIOR, A. S.; ANDRADE, A. C Coeficiente de cultura do capim Tanzânia nos tabuleiros litorâneos do Piauí. In: Congresso Brasileiro de Agrometeorologia, 16., 2009, Belo Horizonte. Anais... Belo Horizonte, 2009.

SANCHES, A. C.; SOUZA, D. P.; JESUS, F. L. F.; MENDONÇA, F. C.; MAFFEI, R. G. Consumo de água de forrageiras tropicais no período de formação de pastagem. Revista Brasileira de Agricultura Irrigada, Fortaleza, v. 11, n. 2, p. 1291-1301, 2017. DOI: http://dx.doi.org/10.7127/rbai.v11n200606

SANTOS, R. F.; CARLESSO, R. Déficit hídrico e os processos morfológicos e fisiológicos das plantas. Revista Brasileira de Engenharia Agrícola e Ambiental, v. 2, n. 3, p. 287-294, 1998. DOI: http://dx.doi.org/10.1590/18071929/agriambi.v2n3p287-294

SANTOS, P. M.; CRUZ, P. G.; ARAUJO, L. C.; PEZZOPANE, J. R. M.; VALLE, C. B.; PEZZOPANE, C.G. Response mechanisms of Brachiaria brizantha cultivars to water déficit stress. Revista Brasileira de Zootecnia, Viçosa, v. 42, n. 11, p. 767-773, 2013. DOI: http://dx.doi.org/10.1590/S1516-35982013001100001

SAS INSTITUTE INC. SAS ${ }^{\circledR}$ 9.4. Statements: Reference. Cary, NC: SAS Institute Inc, 2013.

SILVA, A. R. A.; BEZERRA, F. M. L.; FREITAS, C. A. S.; AMORIM, A. V.; CARVALHO, L. C. C.; PEREIRA FILHO, J. V. Coeficientes de sensibilidade ao déficit hídrico para a cultura do girassol nas condições do semiárido cearense. Revista Brasileira de Agricultura Irrigada, Fortaleza, v. 8, n. 1, p. 38-51, 2014. DOI: http://dx.doi.org/10.7127/rbai.v8n100185

SOIL SURVEY STAFF. Keys to Soil Taxonomy. 12th ed. USDA-Natural Resources Conservation Service, Washington, DC, 2014, 372p

TAIZ, L.; ZEIGER, E. Fisiologia vegetal. 5. ed. Porto Alegre: Artmed, 2013. $918 \mathrm{p}$.

THORNTHWAITE, C. W.; MATHER, J. R. The water balance. Centerton, NJ: Drexel Institute of Technology Laboratory of Climatology, 1955, 104 p. (Publications in Climatology, vol. VIII, n.1)

WOLFINGER, R. D. Covariance structure selection in general mixed models. Communications in Statistics: Simulation Computation, New York, v. 22, n. 4, p. 1079-1106, 1993. DOI: https://dx.doi.org/10.1080/03610919308813143 